\title{
Precision/personalized aneurysm treatment in era of increasing options
}

\author{
Ketan R. Bulsara ${ }^{1}$ \\ Received: 30 July 2018 / Accepted: 31 July 2018 / Published online: 14 August 2018 \\ (C) Springer-Verlag GmbH Austria, part of Springer Nature 2018
}

I congratulate the authors in the management of this unusual problem that arose from stent-assisted coiling of a right middle cerebral artery aneurysm. There is no doubt, based on the provided imaging, that the aneurysm cannot be treated with simple coiling and requires an adjunct such as stenting. In the management of their complication, the authors demonstrate the vast tools available in their armamentarium to manage cerebrovascular diseases. Amongst those discussed in the paper in the management of this patient are innovative clip wrapping of the stent perforation and bypass. If one takes a step back and reassesses this aneurysm, we have a right middle cerebral artery aneurysm that with the demonstrated expertise by the author in this paper could probably easily be treated with microsurgical clipping. Instead, we have a novel complication that is managed with an innovative microsurgical strategy while on antiplatelet therapy. Though I congratulate the authors on the management of this complication, I have reservations in the decision making in proceeding with stentassisted coiling of this aneurysm.

With increasing microsurgical and endovascular treatment options for aneurysm treatments, it becomes even more imperative that the treatment option minimizes patient risk and optimizes outcomes. Though the authors through their expert management of this complication were able to afford this patient a good outcome, I worry that good outcomes such as these in the setting of a potentially devastating complication are likely to be rare. There used to be a time when we only had one option available for the treatment of aneurysms, specifically microsur-

This article is part of the Topical Collection on Vascular NeurosurgeryAneurysm

Ketan R. Bulsara

krbulsara@gmail.com

1 University of Connecticut, Farmington, CT 06030, USA gical treatment. We are now in the era where there are multiple options available. For our patients, it is critical to personalize the treatment of the aneurysm in the context of all available treatment options. To not do so truly deprives our patients of optimal outcomes and threatens the future of all of cerebrovascular surgery. It is also important to restate that the International Subarachnoid Trial results supported that aneurysms amenable to both endovascular and microsurgical treatments fared better with endovascular treatment [1]. The trial did not establish superiority for all aneurysms though it has frequently been misinterpreted as that. The ultimate goal is to optimize patient outcomes. It has always been that and will continue to remain the goal. The optimal treatment thus has to be tailored to not only patient aneurysm morphology/location, but also to patient comorbidity.

It is my personal bias that the optimal management of cerebrovascular disease in today's era requires expertise in microsurgery/skull base surgery/endovascular surgery either in one individual or through a multidisciplinary team. Each member of the team should be well versed in each of these treatment options. These options are precisely that, options. They are not specialties in their own right. They are all options in the treatment of a disease process, in this case, an aneurysm. A cerebrovascular neurosurgeon should possess training and expertise in these skill sets. Whether they choose to practice each of these techniques or not is an individual decision; however, having these skill sets is essential so that all viewpoints can be understood in treating these patients in a multidisciplinary fashion. Ultimately, the goal is to optimize patient outcomes. We can all agree that no other goal is acceptable.

\section{Reference}

1. Leung CH et al (2003) The ISAT trial. Lancet 361(9355):430-431 author reply 432 